\title{
Neonatal conjunctivitis caused by Neisseria gonorrhoeae and Chlamydia trachomatis
}

\author{
ELISABETH REES AND I. ANNE TAIT \\ From the Department of Venereology, Liverpool Royal Infirmary \\ D. HOBSON, RUTH E. BYNG, AND F. W. A. JOHNSON \\ From the Department of Medical Microbiology, University of Liverpool
}

SUMMARY In a selected group of 103 babies referred with neonatal conjunctivitis Neisseria gonorrhoeae was isolated from 11 and Chlamydia trachomatis from 33. Concurrent infection was present in three. One toddler sibling developed chlamydial conjunctivitis. After treatment $C$. trachomatis was re-isolated from six babies during the follow-up period. The discharge started one to three days after delivery in only three babies with gonococcal conjunctivitis and at five to eight days in eight babies. One baby was delivered by caesarean section. $N$. gonorrhoeae was isolated from four asymptomatic fathers, all of whom had urethritis. The mean onset of discharge in the 33 babies from whom Chlamydia was isolated was $7 \cdot 1$ days. One baby was delivered by caesarean section. Chlamydial conjunctivitis was associated with a high incidence of prematurity and of postpartum infection in the mother. Ten fathers of Chlamydia-positive babies were examined. C. trachomatis was isolated from four, all of whom were asymptomatic but had low-grade urethritis. These findings confirm the pathogenic role of $C$. trachomatis in the cervix and indicate the importance to the family of an adequate microbiological investigation of neonatal conjunctivitis.

\section{Introduction}

Infants often develop mild inflammation of the eye a few days after birth. Usually this is due to trauma or transient infection and resolves quickly without treatment. However, more severe forms of purulent conjunctivitis of the neonate are seen and some fail to respond to routine antibiotic treatment.

It has long been recognised that neonatal conjunctivitis could follow intrapartum contamination of the eye with cervical secretion if the mother had a previously unrecognised and untreated genital infection acquired by sexual contact. In the late nineteenth century gonorrhoea was undoubtedly the commonest cause of this condition as well as being the easiest causative organism to identify. As early as 1909, however, Halberstaedter and von Prowazek recognised an abacterial form of infection, and inclusion bodies were identified in the epithelial cells of the infant's conjunctiva and in cellular

Address for reprints: Dr E. Rees, Consultant Venereologist, Department of Venereology, Liverpool Royal Infirmary, Pembroke Place, Liverpool L3 5PU

Received for publication 7 January 1977 scrapings of the genital tract of the parents (Lindner, 1910). This was confirmed by Jones et al. (1959) when it became possible to grow the agent (trachomainclusion conjunctivitis (TRIC) agent, or Chlamydia trachomatis) in the laboratory in chick embryos. More recently, $C$. trachomatis has been isolated in 40 to $50 \%$ of cases of non-gonococcal urethritis (NGU) in the male by tissue culture techniques (Dunlop et al., 1972; Oriel et al., 1976). Infection is transmitted sexually to female partners of men with NGU, and C. trachomatis can be isolated from the cervix in up to $37 \%$ of such women (Hilton et al., 1974; Holmes et al., 1975). If left untreated these women can act as chronic reservoirs of infection and can infect more men or their own infants. The increase of NGU in men by $159 \%$ during the past 10 years compared with an increase of only $29 \%$ in gonococcal urethritis (Department of Health and Social Security, 1965, 1976) is probably a measure of the failure to identify and treat these women. Thus it is likely that paediatricians, obstetricians, and general practitioners will see an increasing number of infants with neonatal conjunctivitis. Those caused by $C$. trachomatis do not respond to conventional treatment with eye 
drops of neomycin, chloramphenicol, gentamicin, or penicillin and persistent infection may result. Some cases progress to palpebral scarring and micropannus similar to, although less in degree than, the sequelae of tropical trachoma (Freedman et al., 1966; Mordhorst and Dawson, 1971). The eyesight is rarely affected.

Infection of the infant with sexually transmissable agents such as $N$. gonorrhoeae or $C$. trachomatis implies that the mother had a cervical infection at the time of birth and that the father may have a persistent but unrecognised urethritis. N. gonorrhoeae can cause pelvic infection and an association between pelvic infection and chlamydial cervicitis has been suggested (Weström, 1975; Märdh et al., 1977). A high incidence of postpartum infection in mothers of infants with chlamydial conjunctivitis has been reported (Dunlop et al., 1966; Rees et al., 1977a). Thus failure to establish quickly the aetiology of neonatal conjunctivitis may have serious sequelae in the mother. The purpose of this paper is to present the results of an investigation into the clinical patterns of neonatal conjunctivitis caused by gonococci and Chlamydia, and the clinical and microbiological investigation of the parents.

\section{Patients and methods}

One hundred and three babies and three toddlers were examined between October 1973 and July 1976. Ninety-five were referred by paediatricians and four by ophthalmologists. Four babies and one toddler sibling were examined because their mothers had been treated for chlamydial cervicitis, three during and one before pregnancy. Two toddlers with conjunctivitis were investigated as contacts of a neonatal sibling. Seven were seen in children's hospitals, four in an eye hospital, 74 in maternity units, and 21 attended as outpatients.

The group forms a selected series of cases of conjunctivitis referred mainly because of failure to respond to routine treatment or because no bacterial growth was reported by the laboratory. A few cases were seen because of unusual presentation or a history of sexually transmitted disease (STD) in the mother.

Specimens were obtained with cottonwool-tipped swabs from the mucosal surface of the lower lids in all cases and from the upper lids if possible, and were examined microscopically and by culture for gonococci, other bacteria, and for candida species. Stuart's medium was used for transport of bacteria but most swabs for gonococcal culture were inoculated directly on to a selective medium containing antibiotics (Lab M).
Swabs for chlamydial culture were kept at $4^{\circ} \mathrm{C}$ in transport medium until they were inoculated into tissue culture between 12 and 24 hours later (Hobson et al., 1974). Immediately before inoculation the swab contents were homogenised in the $2.5 \mathrm{ml}$ of transport medium: $0.4 \mathrm{ml}$ aliquots were inoculated on to each of two monolayer coverslip cultures (MCC) of McCoy cells in $1 \mathrm{oz}$ Universal bottles and centrifuged at $2400 \mathrm{~g}$ for one hour. After 48 hours' incubation at $37^{\circ}$ the MCC were Giemsa-stained and examined by light and dark ground microscopy for intracytoplasmic chlamydial inclusions. Details of the methods have already been described (Johnson, 1975; Johnson and Hobson, 1976). In all cases the results described below represent the direct inoculation of the specimen into tissue culture. No second passage from tissue culture to tissue culture was performed to avoid any risk of false positive results by cross-contamination, and known negative material was inoculated, centrifuged, incubated, and stained with each batch of MCC. All specimens were given serial numbers on arrival in the laboratory and examined in random order.

Specimens for identification of herpes simplex virus by electron microscopical examination and culture were taken in some cases.

Mothers were investigated in the maternity unit if possible, but mothers of premature babies had usually been discharged; these, and mothers whose babies had late onset conjunctivitis, were requested to attend as outpatients if either gonococci or Chlamydia was isolated from the infant. All were investigated for syphilis, gonorrhoea, chlamydial cervicitis, trichomoniasis, candidiasis and, in a few cases, genital herpes by conventional methods. If possible, fathers were similarly investigated if the infant and/or mother had gonococcal, chlamydial, or syphilitic infection.

\section{Results}

$N$. gonorrhoeae was isolated from 11 babies and C. trachomatis from 33 babies and one toddler. Concurrent gonococcal and chlamydial infection was present in three babies. In one case, herpes-like particles were identified by electron microscopy, but not grown in culture.

\section{GONOCOCCAL CONJUNCTIVITIS}

Ten of the 11 babies were delivered at term, and one was premature (birth weight: $2 \cdot 13 \mathrm{~kg}$ ): seven were diagnosed in the maternity unit, and four were admitted to hospitals after being discharged from the maternity unit (three to an eye hospital and one to a children's hospital). 
The reported onset of exudate varied between one and eight days after birth in those diagnosed in maternity units ( 1 to 3 days in three cases; 6 to 8 days in four cases) and between five and six days in the four cases admitted to eye or children's hospitals.

The day of onset in the concurrent gonococcal and chlamydial infections was the sixth day after birth in two cases and the seventh day in one. Three of the four babies re-admitted to hospital had developed discharge at six days and one at five days. These four infants had been treated with topical neomycin or chloramphenicol by the general practitioner and were not admitted until seven to twelve days after birth-that is, when it became obvious that the treatment was failing: two of them had concurrent gonococcal and chlamydial infection.

No signs were observed until the eighth day in the baby delivered by caesarean section; the mother had been treated with ampicillin for pelvic infection from the second day postpartum. $N$. gonorrhoeae was isolated from a high vaginal swab taken before the start of antibiotic treatment. One baby had developed an eye infection in the maternity unit at six days. Gram-negative intracellular diplococci were reported in the smear but because of the mildness of the clinical signs treatment was delayed until full identification by culture and fermentation tests was received two days later. No change in the signs developed during this period.

\section{Findings in mothers}

All eleven mothers were examined: five were married. $N$. gonorrhoeae was isolated from 10 mothers: no isolate was obtained from one mother already receiving ampicillin for pyrexia in hospital. Five mothers developed postpartum infection: pyrexia (1), infected lochia (1), pelvic infection (3). Three of the five had concurrent gonococcal and chlamydial cervical infection.

$N$. gonorrhoeae was isolated from two other married mothers whose babies were being treated for conjunctivitis with topical chloramphenicol at the time of examination. No isolate was obtained from these babies then or later. The onset of exudate was at three and nine days respectively. One mother had concurrent chlamydial cervicitis and one developed hepatitis during the pregnancy and was hepatitis B antigen positive. One of the two fathers had been treated for gonococcal urethritis, the other was not available for examination.

\section{Findings in fathers}

$N$. gonorrhoeae was found in four of five fathers examined. All were asymptomatic. One other father had been treated for gonorrhoea shortly before the birth and another had been treated for urethral discharge.

\section{CHLAMYDIAL CONJUNCTIVITIS}

C. trachomatis was isolated from 26 babies in maternity units, one in the eye hospital, four in children's hospitals, and two attending as outpatients. An isolate was obtained from the 18month-old sister of a baby who developed conjunctivitis at home aged six days. The baby's condition improved after treatment with chloramphenicol, but signs recurred when she was 16 days old and she was admitted to the children's hospital where $C$. trachomatis was isolated. The toddler developed conjunctivitis four days after the baby had been admitted and was treated for several weeks with eye ointment by the general practitioner. Chlamydia was isolated eight weeks later. One baby was delivered by caesarean section, and developed conjunctival discharge in the special care unit aged 11 days (Rees et al., 1977b).

The onset of discharge varied between three and 13 days after the birth with a mean of $7 \cdot 1$ days, or 6.7 days if one excludes three cases given a five-day course of cloxacillin and penicillin from birth, in whom the onset was delayed to 9,11 , and 13 days respectively (Table).

Table Onset of discharge in 33 cases of chlamydial conjunctivitis

\begin{tabular}{lllllllll}
\hline & \multicolumn{1}{c}{ Day of onset } \\
\cline { 2 - 9 } & 3 rd & 5 th & 6 th & 7 th & 8 th & 9 th & 11 th & 13 th \\
\hline No. of cases & 1 & 5 & 11 & 7 & 3 & 2 & 2 & 2 \\
\hline
\end{tabular}

Sixteen infants $(48.8 \%)$ were referred for investigation within 10 days of birth: $26(78.8 \%)$ were referred within 13 days. Isolates were obtained at 17, 19, 20, 24 (2), 30, and 57 days in the remainder. Those in whom referral had been delayed had been treated with topical chloramphenicol with improvement followed by recurrence on cessation of treatment.

All babies developed oedema of the lids and a purulent discharge, which was blood-stained in two cases. Mucosal oedema was present in all cases and was associated with a localised bubbling appearance in the more severe cases and in those in whom the request for investigation was delayed, Fig. 1 a, b. Topical chloramphenicol modified the picture. Discharge often ceased, but mucosal oedema persisted in varying degrees (Fig. 2 a, b, and Fig. 3 a, b). Neither neomycin nor gentamicin had any effect. 

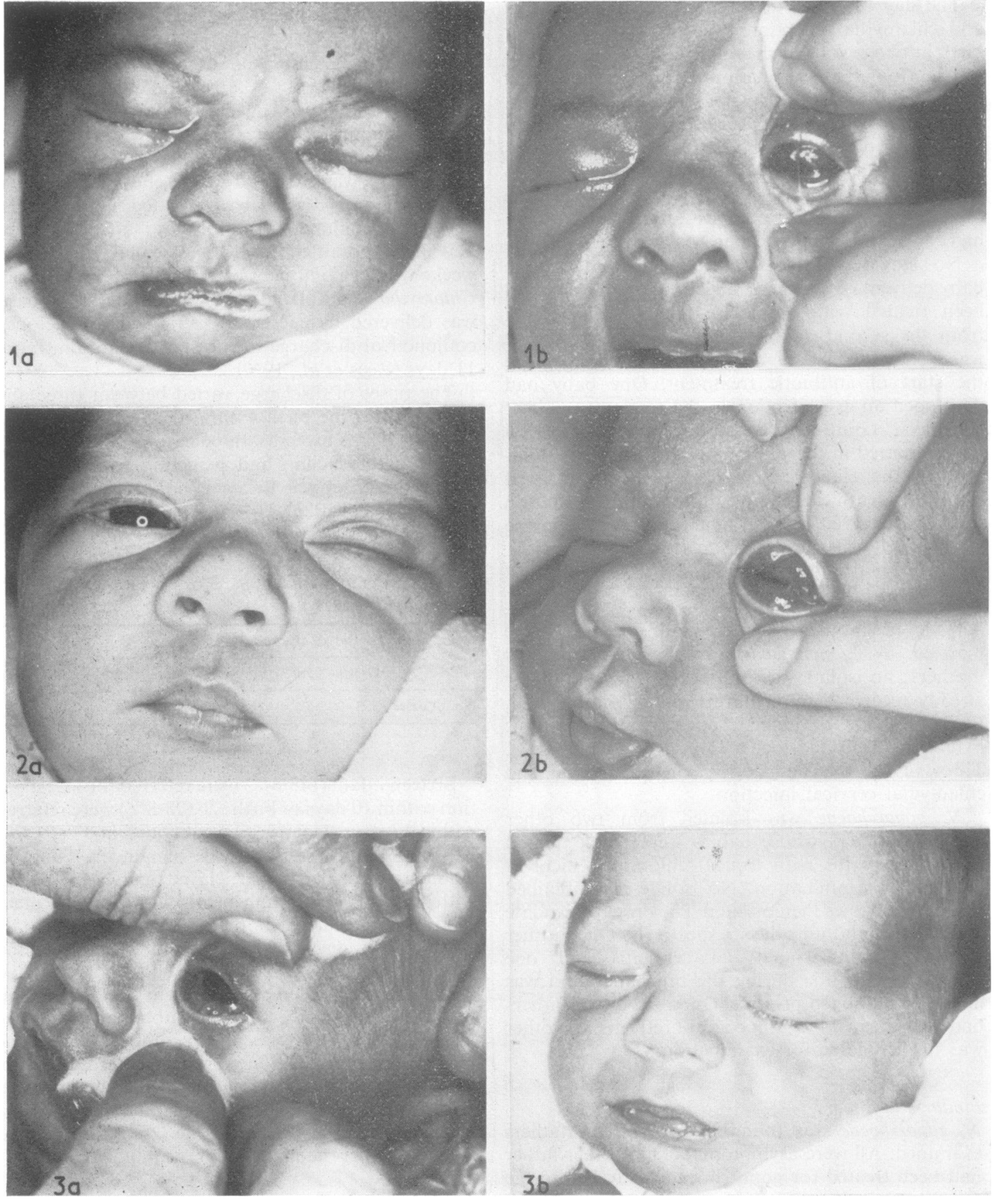
Results of treatment of chlamydial conjunctivitis

After treatment with tetracycline eye ointment prescribed for four weeks, $C$. trachomatis was re-isolated from six of 24 babies who attended for follow-up. Two of these babies were inadequately treated for only two and three weeks. After four weeks' treatment, Chlamydia was re-isolated within a further four weeks in three cases, one of which was the baby born by caesarean section. Chlamydia was re-isolated from another baby four weeks after treatment in hospital and again five months after being treated again for six weeks with topical tetracycline. In five cases minor symptoms and signs recurred on relapse.

Fourteen babies were premature $(42 \cdot 4 \%)$. The gestation period was between 30 and 34 weeks in 10 and between 35 and 37 in four, birth weight was between 1.5 and $2.7 \mathrm{~kg}$. Excluding the four babies examined as contacts because their mothers had been treated for chlamydial cervicitis before or during the pregnancy, the incidence of prematurity in the group from whom neither gonococci nor Chlamydia was isolated was nine of 58 cases $(15 \cdot 5 \%)$. The difference in the incidence of prematurity in chlamydial positive and negative babies is significant $\left(\%^{2}=6.7 \mathrm{P}<0.01\right)$

Findings in mothers of babies with chlamydial conjunctivitis

Nineteen of the 33 mothers were married; 31 were examined. One specimen was cytotoxic: Chlamydia was isolated from the cervix in 20 of the remaining 30 mothers. Seven of the 10 negative mothers were

Fig. 1 Conjunctivitis aged 7 days. Treated with neomycin eye drops for two days without response. C. trachomatis isolated aged nine days.

(a) Palpebral oedema. (b) Severe mucosal oedema with bubbling and mucopurulent discharge.

Fig. 2 Conjunctivitis aged 5 days. Treated with neomycin eye drops for two days without response. Chloramphenicol eye drops for five days; discharge ceased, palpebral oedema lessened. C. trachomatis isolated aged 12 days.

(a) Persisting but lessening palpebral oedema indicated by slight wrinkling of skin. (b) Well-marked mucosal oedema with bubbling on upper lid.

Fig. 3 Conjunctivitis aged 3 days. Streptococcus faecalis isolated. Relapsed within 5 days of completion of each of two 5-day courses of chloramphenicol eye drops. C. trachomatis isolated aged 30 days on fourth day of third course in special care unit.

(a) Minimal palpebral oedema. (b) Mild conjunctivitis mucosal oedema with bubbling. Two possible early follicles. receiving, or had received, antibiotics for postpartum infection. Nineteen of the 31 mothers developed postpartum infection: pelvic infection (11), pyrexia (3), infected lochia (4), wound infection (1).

C. trachomatis was isolated from eight of the 11 mothers with pelvic infection. All three negative mothers had received or were receiving antibiotics at the time of examination. One Chlamydia-positive mother with pelvic infection had been treated with ampicillin for infected lochia before discharge from the maternity unit. Another mother of a premature baby gave a history of severe lower abdominal pain three weeks before she attended as an outpatient, but no tenderness was found on pelvic examination and she is, therefore, excluded from the group. Sera were examined for psittacosis lymphogranuloma venereum group antibody in nine of the 11 cases and all had levels of 1:20 or higher with a rising titre in early cases. Titres of $1: 80$ rising to $>1: 1280$ were reported in two cases. (Full details of group antibody levels will be reported elsewhere.)

\section{Findings in fathers}

C. trachomatis was isolated from four of 10 fathers examined. All were asymptomatic but low grade urethritis was found. One normal father had recently taken antibiotics.

\section{Discussion}

The results of this investigation suggest that neonatal eye infection caused by sexually transmissable organisms is common. This infection is unlikely to lead to impairment of sight unless neglected, but failure to establish the cause before treatment may have serious consequences to the mother in whom risk of postpartum extension of infection from the cervix appears to be high. Furthermore, the woman can be reinfected by the untreated asymptomatic man and both may be a source of infection to other members of the family.

A surprising feature of this group of babies is the late onset of gonococcal conjunctivitis. Only three of the 11 babies developed discharge within three days of birth and for this reason the diagnosis was made after the baby had been discharged from the maternity unit in four cases. It is, of course, possible that infection was not intrapartum but followed contact with infected hands or towels. Nevertheless, in one case of mild conjunctivitis developing in hospital at six days no increase in the slight conjunctival discharge occurred in the 48 hours during which confirmation of the positive Gram-stained smear was awaited. The baby delivered by caesarean section developed conjunctivitis in hospital at eight days. His mother started taking antibiotics 48 hours 
postpartum and was, therefore, non-infectious from that time. (Gonococci were not isolated again.) These cases suggest that gonococcal conjunctivitis may in some cases have a comparatively long incubation period and may not be acute. Alternatively, frequent postpartum transmission of infection to the eyes of infants must be postulated. Gonococcal conjunctivitis has in the past responded promptly to topical and systemic treatment with penicillin. Difficulties in treatment may be encountered in the future owing to infections with $\beta$-lactamase producing strains of gonococci which have been identified in Liverpool since February: 1976 (Percival, et al., 1976).

Because of the late onset of chlamydial conjunctivitis (six to seven days after birth) most infected babies will be seen by the general practitioner or at the baby clinic. The high incidence of premature babies in this series may simply mean that they are more likely to be diagnosed than full-term babies because they remain in hospital for a longer time than the incubation period of the infection. However, the possibility that chlamydial cervicitis might involve the membranes overlying the internal os and result in premature rupture has been suggested (Rees et al., 1977b). Some support is given to this suggestion by this investigation in which a significant difference in the prematurity rate for Chlamydia-positive and Chlamydia-negative babies was found. Thus the association between prematurity and conjunctivitis may be causal-that is, the mother's chlamydial infection may be responsible for both conditions as well as for an increased chance that she may develop postpartum infection.

In an epidemiological study, Watson and Gairdner (1968) reported four definite and four suggestive cases of chlamydial infection in 44 cases of neonatal conjunctivitis screened for inclusions in a maternity unit. Exudate was often scanty and not obviously purulent : inflammation and palpebral oedema were negligible. No attempt was made to culture the agent. No specific treatment was given but in two of the three cases followed for three to 24 weeks postpartum there was scarring of the conjunctiva, and one of the two had a continuing and active pannus. Such cases would not have been referred for investigation in our selected series in which all babies had purulent conjunctivitis with well-marked mucosal oedema and, in untreated cases, palpebral oedema.

Topical neomycin does not inhibit Chlamydia and has a broad antibacterial spectrum. It is the first line treatment of choice in non-gonococcal conjunctivitis and babies who fail to respond should be investigated for Chlamydia. Inadequate treatment with chloramphenicol or short courses of tetra- cycline may suppress but not eradicate Chlamydia. Palpebral oedema and conjunctival exudate may clear but conjunctivitis often persists. The importance of everting the lids to examine the conjunctiva of all babies treated for neonatal conjunctivitis and as part of the general examination of babies before discharge from the maternity unit is clearly indicated.

The isolation of Chlamydia from the 18-month-old sister of one infant admitted to hospital with severe purulent conjunctivitis suggests eye to eye transmission such as occurs in hyperendemic trachoma (Jones, 1975; Grayston et al., 1977).

The asymptomatic nature of both gonococcal and chlamydial infection in eight fathers underlines the necessity for early accurate diagnosis of neonatal conjunctivitis. Only if gonococci or Chlamydia are isolated will a full family investigation be carried out and the risk of pelvic infection in the mother be reduced by early diagnosis and treatment. The fact that nearly $60 \%$ of the mothers in this series were married possibly reflects the degree of extramarital contact occurring at the present time.

This work was supported by a project grant from the Medical Research Council.

We are indebted to Professor F. Harris and Dr Anne McCandless for referring cases: to $\mathrm{Mr} \mathrm{A}$. Patterson for ophthalmological review of treated cases: to Dr A. Percival and Dr R. E. Rewell for bacteriological investigations: to $\mathrm{Dr}$ Bruce White for viral studies and psittacosis/LGV group antibody tests: to Dr G. C. Turner for serological tests for syphilis and to Mr M. C. K. Tweedie for statistical analysis.

Our junior colleagues, Dr A. K. Ghosh, Dr Jean C. Davies, Dr P. B. Carey, and Dr Joyce Angami gave invaluable assistance.

The photographs were taken by Mr P. J. Nixon, the University Department of Obstetrics and Gynaecology.

\section{References}

Department of Health and Social Security (1965). Annual Report of the Chief Medical Officer for the Year 1964. HMSO: London.

Department of Health and Social Security (1976). Annual Report of the Chief Medical Officer for the Year 1974. HMSO: London.

Dunlop, E. M. C., Al-Hussaini, M. K., Freedman, A., Garland, J. A., Harper, I. A., Jones, B. R., Race, J. W., du Toit, M. S., Treharne, J. D., and Wright, D. J. M. (1966). Infection by TRIC agent and other members of the Bedsonia group; with a note on Reiter's disease. III. Genital infection and disease of the eye. Transactions of the Ophthalmological Societies of the United Kingdom, 86, 321-334.

Dunlop, E. M. C., Vaughan-Jackson, J. D., Darougar, S., and Jones, B. R. (1972). Chlamydial infection. Incidence in non-specific urethritis. British Journal of Venereal Diseases, 48, 425-428.

Freedman, A., Al-Hussaini, M. K., Dunlop, E. M. C., Emarah, M. H. M., Garland, J. A., Harper, I. A., Jones, B. R., Race, J. W., du Toit, M. S., Treharne, J. D., and Wright, D. J. M. (1966). Infection by TRIC agent and other members of the Bedsonia 
group; with a note on Reiter's disease. II. Ophthalmia neonatorum due to TRIC agent. Transactions of the Ophthalmological Societies of the United Kingdom, 86, 313-320.

Grayston, J. T., Yeh, L., Wang, S., Kuo, C., Beasley, R. P., and Gale, J. L. (1977). Pathogenesis of ocular Chlamydia trachomatis infections in humans. In Symposium on Non-gonococcal Urethritis and Related Oculo-genital Infections, 28-30 April 1976, Lake Placid, New York. American Society of Microbiology: Washington DC.

Halberstaedter, L., and von Prowazek, S. (1909). Ueber chlamydozoenbefunde bei blennorrhoea neonaturum non-gonorrhoica. Berliner klinische Wochenschrift, 46, 1839-1840.

Hilton, A. L., Richmond, S. J., Milne, J. D., Hindley, F., and Clarke, S. K. R. (1974). Chlamydia $A$ in the female genital tract. British Journal of Venereal Diseases, 50, 1-10.

Hobson, D., Johnson, F. W. A., Rees, E., and Tait, I. A. (1974). Simplified method of diagnosis of genital and ocular infections with Chlamydia. Lancet, 2, 555-556.

Holmes, K. K., Handsfield, H. H., Wang, S. P., Wentworth, B. B., Turck, M., and Alexander, E. R. (1975). Etiology of non-gonococcal urethritis. New England Journal of Medicine, 292, 1195-1205.

Johnson, F. W. A. (1975). A comparison of staining techniques for demonstrating group A Chlamydia in tissue culture. Medical Laboratory Technology, 32, 233-238.

Johnson, F. W. A., and Hobson, D. (1976). Factors affecting the sensitivity of replicating McCoy cells in the isolation and growth of Chlamydia A (TRIC agents). Journal of Hygiene, 76, 441-451.

Jones, B. R. (1975). The prevention of blindness from trachoma. Transactions of the Ophthalmological Societies of the United Kingdom, 95, 16-33.

Jones, B. R., Collier, L. H., and Smith, C. H. (1959). Isolation of virus from inclusion blennorrhoea. Lancet, 1, 902-905.
Lindner, K. (1910). Zur aetiologie der gonokokkenfrien urethritis. Wiener klinische Wochenschrift, 23, 283-284.

Märdh, P. A., Ripa, T., and Weström, L. (1977). In Symposium on Non-gonococcal Urethritis and Related Oculo-genital Infections, 28-30 April 1976, Lake Placid, New York. American Society of Microbiology: Washington DC.

Mordhorst, C. H., and Dawson, C. (1971). Sequeae of neonatal inclusion conjunctivitis and associated disease in parents. American Journal of Ophthalmology, 71, 861-867.

Oriel, J. D., Reeve, P., Wright, J. T., and Owen, J. (1976). Chlamydial infection of the male urethra. British Journal of Venereal Diseases, 52, 46-51.

Percival, A., Rowlands, J., Corkill, J. E., Alergant, C. D., Arya, O. P., Rees, E., and Annels, E. H. (1976). Penicillinase-producing gonococci in Liverpool. Lancet, 2, 1379-1382.

Rees, E., Tait, I. A., Hobson, D., and Johnson, F. W. A. (1977a). Chlamydia in relation to cervical infection and pelvic inflammatory disease. In Symposium on Non-gonococcal Urethritis and Related Oculo-genital Infections, 28-30 April 1976, Lake Placid, New York. American Society of Microbiology: Washington DC.

Rees, E., Tait, I. A., Hobson, D., and Johnson, F. W. A. (1977b). Perinatal chlamydial infections. In Symposium on Non-gonococcal Urethritis and Related Oculo-genital Infections, 28-30 April 1976, Lake Placid, New York. American Society of Microbiology: Washington DC.

Watson, P. G., and Gairdner, D. (1968). TRIC agent as a cause of neonatal eye sepsis. British Medical Journal, 3, 527-528.

Weström, L. (1975). Effect of acute pelvic inflammatory disease on fertility. American Journal of Obstetrics and Gynecology, 121, 707-713. 